\title{
Evaluation of Diagnostic Performance of Screening Thyroid Ultrasonography and Imaging Findings of Screening-Detected Thyroid Cancer
}

\section{Jeongin Yoo, $M D^{1}$ \\ Hye Shin Ahn, MD ${ }^{1}$ \\ Soo Jin Kim, MD² \\ Sung Hee Park, MD \\ Mirinae Seo, MD',3 \\ Semin Chong, MD ${ }^{1}$}

\author{
${ }^{1}$ Department of Radiology, Chung-Ang \\ University Hospital, Chung-Ang University \\ College of Medicine, Seoul, \\ ${ }^{2}$ Department of Radiology, New Korea \\ Hospital/Human Medical Imaging and \\ Intervention Center, Gimpo, \\ ${ }^{3}$ Department of Radiology, \\ Kyung Hee University Hospital, \\ Kyung Hee University College of Medicine, \\ Seoul, Korea
}

\begin{abstract}
Purpose
The purpose of this study was to evaluate the diagnostic performance and cost of screening thyroid ultrasonography (US) in an asymptomatic population and determine the US features of screening-detected thyroid cancer.
\end{abstract}

\section{Materials and Methods}

This study included 1,845 asymptomatic participants who underwent screening thyroid US between March and August 2012 at the screening center in our hospital. We evaluated the diagnostic performance of screening thyroid US for thyroid cancer and the average cost of diagnosis for each patient. We also determined the characteristic US features of screeningdetected thyroid cancer.

\section{Results}

Of the 1,845 subjects, 661 showed no abnormalities, 1,155 exhibited benign thyroid nodules, and 29 exhibited thyroid cancer. Imaging features such as solid composition, hypoechogenicity, taller-than-wide axis, and ill-defined or spiculated margins of nodules were suggestive of malignancy. The rate of detection of cancer was $1.6 \%(29 / 1,845)$, and the sensitivity, specificity, and positive and negative predictive values were $100 \%(18 / 18)$, $98.7 \%$ (1,051/1,065), 56.3\% (18/32), and 100\% (1,051/1,051), respectively. Of 18 patients who underwent thyroidectomy, three (16.7\%) had a pathological tumor staging of $\mathrm{T} 3$, and four (22.2\%) had a pathological nodal staging of N1a. The average cost of diagnosis for each patient with cancer was $\$ 7,319$.

\section{Conclusion}

Screening thyroid US exhibited a good diagnostic performance, with a feasible social cost of use. This modality demonstrated significant differences in sonographic features between screening-detected cancer and benign nodules.

\section{College of Medicine, 102 Heukseok-ro,}

Dongjak-gu, Seoul 06973, Korea

Tel: 82-2-6299-3230

Fax: 82-2-6299-3231

E-mail: ach0224@gmail.com

\section{Key words}

Costs and cost analysis, Diagnostic techniques and procedures, Thyroid neoplasms, Ultrasonography

\section{Introduction}

Thyroid nodules occur very commonly and are diagnosed in $4 \%$ of adults by palpation, $67 \%$ by ultrasonography (US), and $50 \%$ by pathological examination at autopsy [1-3]. Recently, the introduction of high resolution US as a screening tool for thyroid cancer has made it possible to detect nodules less than $1 \mathrm{~cm}$ in size [4-6]. Moreover, the incidence of thyroid cancer has reportedly increased in different parts of the world over the past several decades [7]. Kilfoy et al. [7] found that the recent increase in thyroid cancer appears to be neither restricted to a particular region of the world nor influenced by the underlying rates of thyroid cancer. However, whether the increased incidence of thyroid cancer is real or a result of over-diagnosis from thyroid screening is still a matter of controversy. Some physicians have attributed this increase in incidence to increased detection of small cancers $(<1 \mathrm{~cm}$ in size) because of the increase in application of high-resolution US for screening [8,9]. Davies and Welch [8] 
suggested that, despite the gradual increase in the incidence of thyroid cancer in the United States, cancer mortality rates have been stable over several decades because of early diagnosis of papillary microcarcinomas. However, other physicians have expressed the opposite opinion [10-12]. For example, Morris and Myssiorek [11] reported that the significant increase in the incidence of large cancers and cancers with clinically significant adverse pathological features was due not only to increased screening, but also to an actual increase in cancer incidence.

Reflecting the international trend, the incidence of thyroid cancer in South Korea has been increasing rapidly since the turn of the century. The rate of diagnosis of thyroid cancer in 2011 was 15 times that observed in 1993, and it is now the most commonly diagnosed type of cancer [13]. The government has provided national health insurance to 50 million citizens since the 1980s, including a national screening program for cancer. Although thyroid cancer screening has not been included in the program, many self-referred patients frequently choose to include US screening as an inexpensive add-on for about $\$ 100$. Earlier in 2015, a few Korean physicians expressed concern regarding the over-diagnosis of thyroid cancer through screening programs [9]. They asserted that the incidence of thyroid cancer in South Korea had rapidly increased since the 20th century, and that this increase could be attributed to the increased detection of papillary thyroid cancer because of the screening efforts. To address the necessity of guidelines for thyroid cancer screening, the Korean Medical Association recently appointed a multidisciplinary expert committee that conducted a meta-analysis toward this purpose [14]. However, the current evidence was insufficient to assess the balance of advantages and disadvantages of screening US for thyroid cancer, and the committee could not propose a definite standard for the thyroid screening program. As a result, the role of screening thyroid US still remains controversial, and there have been very few studies investigating the screening of thyroid cancer in a medical setting to date $[15,16]$.

The social costs and cost-benefits of screening thyroid US have also initiated another important debate. Specifically, some physicians have expressed concern regarding the increasing costs of unnecessary examination or surgery because of screening-detected small cancers $[5,6,17]$. This topic, too, remains controversial and has not been evaluated in previous studies to the best of our knowledge. Therefore, the purpose of this study was to evaluate the diagnostic performance and cost-analysis of screening thyroid US in the medical screening center of our hospital and determine the US features of screening-detected thyroid cancer.

\section{Materials and Methods}

\section{Patients}

This retrospective study was approved by the institutional review board of our institution, and the necessity for informed patient consent for research was waived. Informed consent was obtained from all patients prior to US-guided fine-needle aspiration biopsy. A total of 1,923 consecutive asymptomatic and self-referred subjects underwent screening thyroid US at the medical screening center of our hospital between March and August 2012, of whom, 1,845 were included in this study. We excluded 18 patients with a history of thyroid surgery as well as 60 patients who were diagnosed with thyroid nodules on screening US but had an insufficient follow-up period ( $<1$ year). The mean follow-up duration was 495 days (range, 365 to 730 days) in cases in which nodules were detected in the initial screening US.

\section{US imaging and US-guided fine-needle aspiration}

Thyroid US examination was performed by one of two board-certified radiologists at the medical screening center of our hospital using high-resolution US equipment (LOGIQ 7 and LOGIQ E9, GE Healthcare, Milwaukee, WI and IU 22, Philips Ultrasound, Bothell, WA) with a $5-12 \mathrm{MHz}$ linear transducer. According to the Consensus Statement and Recommendation of the Korean Society of Thyroid Radiology (KSThR), thyroid nodules were categorized into three groups according to their ultrasonographic features, suspicious malignant, probably benign, and indeterminate nodules (Table 1) [18]. Suspicious nodules were defined by the presence of at least one of five suspicious findings (taller-thanwide shape, spiculated margin, marked hypoechogenicity, or micro/macrocalcifications). In contrast, simple cysts or cystic (>90\% of the cystic portion) or predominantly cystic (>50\% of the cystic portion and $\leq 90 \%$ of the cystic portion) nodules with reverberating artifacts and spongiform nodules were defined as probably benign. An indeterminate nodule was defined when a nodule was not classifiable as probably benign or malignant. After screening US, patients with suspicious malignant nodules, indeterminate nodules $>1 \mathrm{~cm}$ in size, probably benign nodules $>2 \mathrm{~cm}$ in size (except simple cysts), or nodules exhibiting growth (20\% increase in nodule diameter, with a minimum increase of at least $2 \mathrm{~mm}$ in two or more dimensions) on follow-up US examinations were referred to the department of radiology. One of four subspecialty-trained thyroid radiologists with at least 2 years of thyroid imaging experience performed US-guided fine-needle aspiration (FNA) according to the indications of the consensus statement of KSThR (Table 1) [18]. Using a 23-gauge nee- 
Table 1. Consensus statement and recommendations of the Korean Society of Thyroid Radiology

\begin{tabular}{|c|c|c|c|}
\hline & Probably benign & Indeterminate & Suspicious for malignancy ${ }^{\mathrm{a}}$ \\
\hline \multirow[t]{5}{*}{ US features } & Spongiform & Not classifiable as benign or malignant & Taller-than-wide shape \\
\hline & Cystic & Hyper/iso/hypoechogenicity & Spiculated margin \\
\hline & $\begin{array}{l}\text { Predominantly cystic or cystic nodule } \\
\text { with reverberating artifacts }\end{array}$ & Ovoid to round or irregular shape & Marked hypoechogenicity \\
\hline & & Smooth or ill-defined margin & Micro/macrocalcifications \\
\hline & & Rim calcification & \\
\hline \multirow[t]{3}{*}{ Management } & $\leq 1 \mathrm{~cm}$, no follow-up & $>1 \mathrm{~cm}, \mathrm{FNA}$ & $>5 \mathrm{~mm}, \mathrm{FNA}$ \\
\hline & $>1 \mathrm{~cm}$, follow-up US & & \\
\hline & $>2 \mathrm{~cm}$, selective FNA & & \\
\hline
\end{tabular}

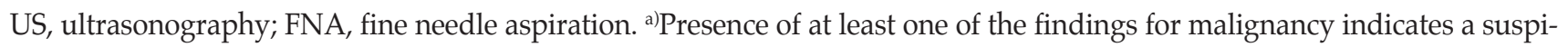
cious malignant nodule.

dle attached to a 5-mL syringe, numerous multi-directional passes were performed through the nodule for successful sampling. The specimens were preserved in bottles with $95 \%$ ethanol for liquid-base cytological examination. Cytopathological reports of FNA were prepared according to the Bethesda system [19]. Repeat FNA was conducted when the cytological findings of nodules were found to be non-diagnostic (ND) or atypia of undetermined significance or follicular lesions of undetermined significance (AUS/FLUS). Repeat FNA or surgical excision was performed when the cytology was reported to be either follicular neoplasm (FN) or suspicious for FN.

\section{Imaging analysis, reference standards, and statistical analysis}

The US features of the nodules were retrospectively reviewed in consensus by four subspecialty-trained thyroid radiologists with 4-10 years of experience. The size, composition, margin, calcification, axis, and echogenicity of each lesion were described based on US findings. In cases in which US findings revealed multiple nodules, the most suspicious nodule was selected for further evaluation. In addition, when US findings revealed multiple similar probably benign or indeterminate nodules, the largest one was selected. The US features of screening-detected thyroid cancer were then compared with those of benign nodules.

The proportion of subjects with malignancy was calculated according to their sex and age. The diagnostic performance (sensitivity, specificity, and positive and negative predictive values) of screening thyroid US for malignancy was then determined.

All patients with thyroid nodules identified by screening US underwent US-guided FNA or received adequate followup for at least 1 year. In cases of malignancies, surgical exci- sion was performed by one of two surgeons with 30 and 10 years of thyroid surgery experience. The specimens from FNA and surgical excision were analyzed by a thyroid pathologist with 15 years of experience.

All statistical analyses were conducted using the commercially available software SPSS ver. 17.0 (SPSS Inc., Chicago, IL). Categorical variables were compared using chi-squared or Fisher exact tests, and continuous variables were compared using the Student's t test. A p-value $<0.05$ was considered to indicate statistical significance.

\section{Results}

A total of 1,845 subjects were included in this study (Fig. 1), including 1,207 women and 638 men. The mean age of the subjects was 48.7 years (range, 19 to 85 years). Among the subjects, $661(35.8 \%)$ exhibited no evidence of thyroid nodule, while $1,184(64.2 \%)$ were found to have single/multiple thyroid nodules upon screening US. Diffuse thyroiditis was observed in 131 of 1,845 subjects on thyroid US (7.0\%). Of the 1,184 subjects with nodules, $1,051(88.8 \%)$ had probably benign nodules, $101(8.5 \%)$ had indeterminate nodules, and $32(2.7 \%)$ had suspicious malignant nodules. All 111 patients with suspicious malignant nodules $(n=32)$, indeterminate nodules $>1 \mathrm{~cm}(\mathrm{n}=60)$, or probably benign nodules that exhibited growth on follow-up examinations $(n=19)$ underwent US-guided FNA at the department of radiology (Figs. 2 and 3). A total of 1,073 patients, including 41 with indeterminate nodules and 1,032 with probably benign nodules, achieved sufficient follow-up duration and exhibited no significant changes in follow-up US examinations. The cytopathological findings of the FNA specimens were as fol- 


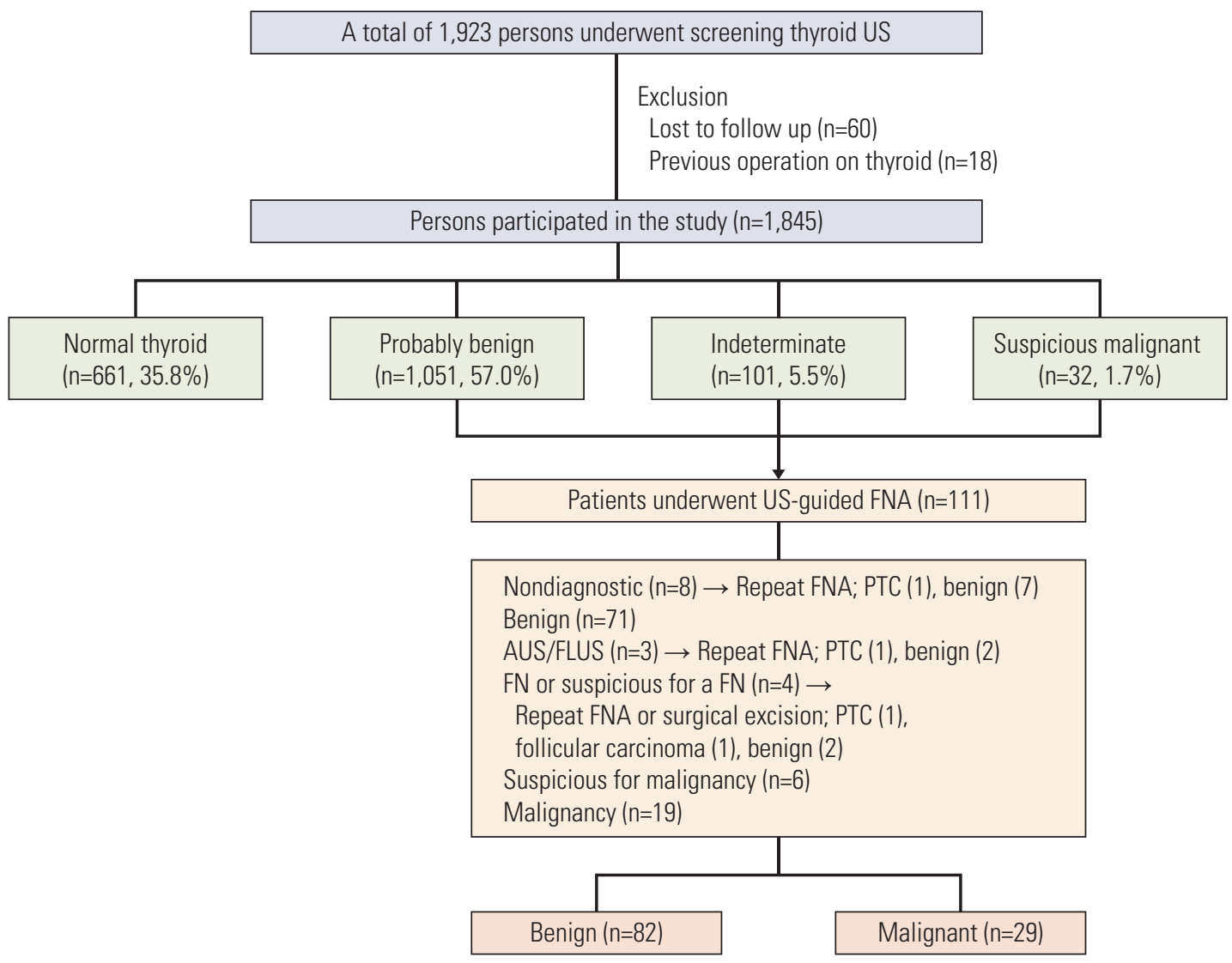

Fig. 1. Flow diagram of the study. US, ultrasonography; FNA, fine-needle aspiration; PTC, papillary thyroid carcinoma; AUS/FLUS, atypia of undetermined significance or follicular lesions of undetermined significance; FN, follicular neoplasm.
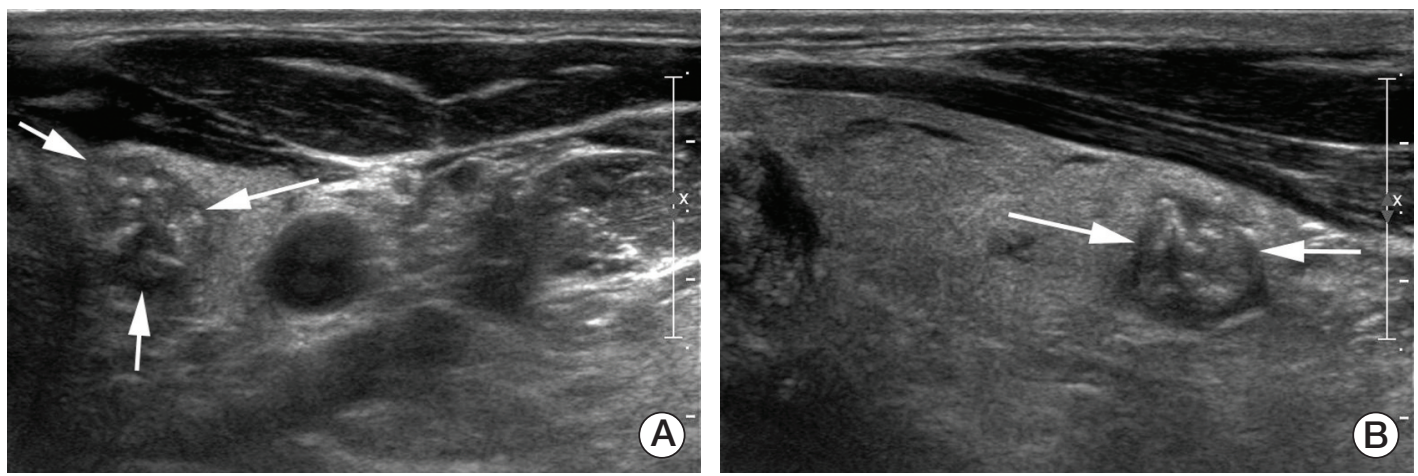

Fig. 2. A 54-year-old man with a $1.2 \mathrm{~cm}$ suspicious malignant nodule in the left thyroid gland. (A, B) Axial and longitudinal ultrasonography images show a hypoechoic nodule with a taller-than-wide axis, ill-defined margin, and multiple hyperechoic foci suggesting micro/macrocalcifications (arrows). Upon ultrasonography-guided fine needle aspiration and pathological evaluation, the nodule was revealed to be a papillary thyroid carcinoma. The patient underwent total thyroidectomy, and the final diagnosis was papillary thyroid carcinoma. 

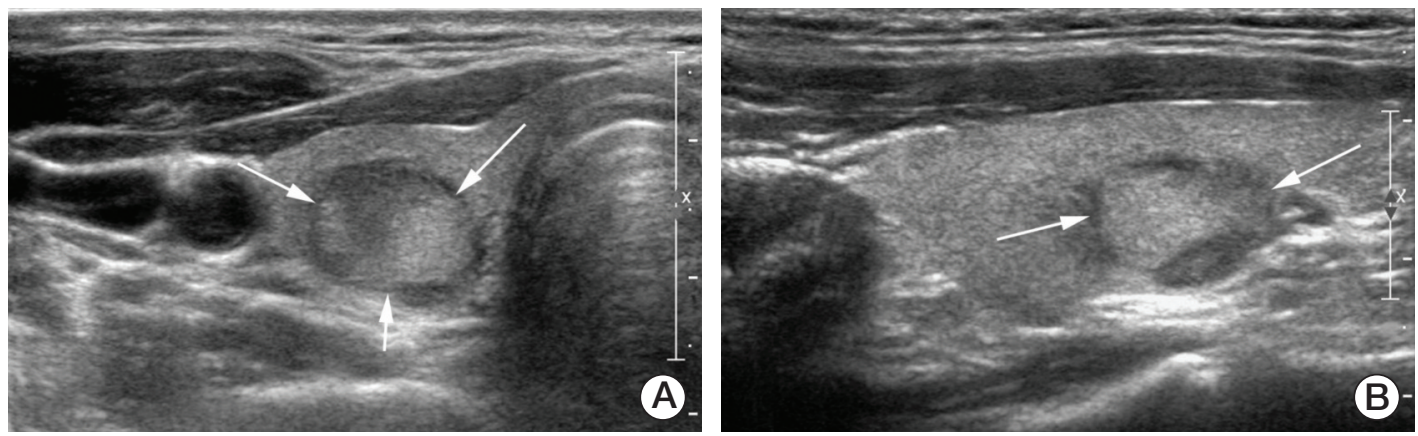

Fig. 3. A 40-year-old man with a 1.3-cm indeterminate nodule in the right thyroid gland. (A, B) Axial and longitudinal ultrasonography (US) images show an oval solid isoechoic nodule with wider-than-tall axis (arrows). Upon US-guided fine needle aspiration and pathological evaluation, the nodule was found to be suspicious for follicular neoplasm. The patient underwent total thyroidectomy, and the final diagnosis was minimally invasive follicular carcinoma (R-minor-2).

Table 2. US features of screening-detected thyroid cancer

\begin{tabular}{|c|c|c|c|c|}
\hline & Benign $(n=1,155)$ & Malignant $(n=29)$ & Total & p-value \\
\hline \multicolumn{5}{|l|}{ Composition } \\
\hline Solid $<50 \%$ & $467(40.4)$ & 0 & 467 & $<0.001$ \\
\hline Solid $>50 \%$ & $688(59.6)$ & $29(100)$ & 717 & \\
\hline \multicolumn{5}{|l|}{ Echogenicity } \\
\hline Hyper/Isoechoic & $82(7.1)$ & 0 & 82 & 0.257 \\
\hline Hypoechoic & $1,073(92.9)$ & $29(100)$ & 1,102 & \\
\hline \multicolumn{5}{|l|}{ Margins } \\
\hline Smooth margin & $1,093(94.6)$ & $3(10.3)$ & 1,096 & $<0.001$ \\
\hline Ill-defined or spiculated margin & $62(5.4)$ & $26(89.7)$ & 88 & \\
\hline \multicolumn{5}{|l|}{ Axis } \\
\hline Wider than tall & $1,129(97.7)$ & $5(17.2)$ & 1,134 & $<0.001$ \\
\hline Taller than wide & $26(2.3)$ & $24(82.8)$ & 50 & \\
\hline \multicolumn{5}{|l|}{ Calcification } \\
\hline Calcification & $930(80.5)$ & $21(72.4)$ & 951 & $<0.001$ \\
\hline No calcification & $225(19.5)$ & $8(27.6)$ & 233 & \\
\hline Size, mean \pm SD $(\mathrm{mm})$ & $6.7 \pm 5.9$ & $8.2 \pm 6.7$ & & \\
\hline$<10$ & $889(77.0)$ & $21(72.4)$ & 910 & 0.512 \\
\hline$\geq 10$ & $266(23.0)$ & $8(27.6)$ & 274 & \\
\hline
\end{tabular}

Values are presented as number (\%) unless otherwise indicated. US, ultrasonography; SD, standard deviation.

lows (Fig. 1): ND, 8; benign, 71; AUS / FLUS, 3; FN or suspicious for a FN, 4; suspicious for malignancy, 6; and malignancy, 19. Repeat FNA was performed for nodules with ND $(n=8)$ and AUS / FLUS ( $n=3)$ diagnoses, which resulted in two patients being diagnosed with papillary thyroid cancer and nine with benign nodules. Of the four patients diagnosed with FN or suspicious for FN, one underwent total thyroidectomy, and the pathological findings of the surgical specimen indicated papillary thyroid cancer. Another patient underwent repeat FNA, which confirmed FN, as well as total thyroidectomy. Analysis of a surgical specimen obtained during the thyroidectomy was confirmed follicular carcinoma. The remaining two patients underwent repeat FNA and were each diagnosed with benign follicular nodule and chronic lymphocytic thyroiditis. Finally, 29 nodules were diagnosed as malignancies and 82 as benign nodules.

Statistical analysis revealed no significant correlations between age and incidence of malignant nodules in either 
women or men $(\mathrm{p}=0.511)$. There were also no statistically significant differences in the rate of malignancy between women and men ( $p=0.925)$. The US features of screeningdetected thyroid cancer were analyzed with respect to composition, echogenicity, margin, axis, calcification, and size. In contrast to benign nodules, malignant nodules tended to be solid, ill-defined or speculated, taller than wide, and calcified. These differences in US features between malignant and benign nodules were statistically significant. There were no significant differences in echogenicity and size between benign and malignant nodules (Table 2).

The prevalence of thyroid incidentalomas, defined as benign or malignant thyroid nodules discovered upon screening US, was $64.2 \%(1,184 / 1,845)$. The rate of malignancy, defined as the number of cancers per number of incidentalomas, was $2.4 \%(29 / 1,184)$. The rate of cancer detection, defined as the number of cancers per total number of US examinations, was $1.6 \%$ (15.7 cancer patients per 1,000 exams; $29 / 1,845)$. Of the 32 patients with suspicious malignant nodules upon initial screening US, 18 were pathologically diagnosed with cancer and 14 with benign nodules. All 1,051 patients diagnosed with probably benign nodules upon initial screening US were finally confirmed as having benign nodules by biopsy ( $\mathrm{n}=19)$ or stable nodules upon follow-up US ( $\mathrm{n}=1,032)$. The sensitivity, specificity, and positive and negative predictive values of screening thyroid US were $100 \%$ (18/18), $98.7 \%$ (1,051/1,065), 56.3\% (18/32), and $100 \%$ $(1,051 / 1,051)$, respectively.

Of the 29 patients with a pathological diagnosis of malignancy, 18 underwent surgery at our hospital and 11 were transferred to other hospitals. Thirteen nodules in these patients were $<1 \mathrm{~cm}$ in size, and the mean size of the nodules was $8.6 \mathrm{~mm}$ (range, 5 to $37 \mathrm{~mm}$ ). According to the final pathological results, 17 patients had papillary thyroid cancer and one had follicular carcinoma. In terms of pathological tumor staging, three patients exhibited T3 $(16.7 \%)$, one exhibited T2 $(5.6 \%)$, and 14 exhibited $\mathrm{T} 1(77.8 \%)$ stages of cancer. In terms of nodal stating, four patients exhibited N1a (22.2\%), 12 exhibited N0 (66.7\%), and two exhibited Nx $(11.1 \%)$ stages of cancer. Among the operative patients, 14 $(77.8 \%)$ exhibited thyroid microcarcinoma, which was defined by nodules with longitudinal diameters $<10 \mathrm{~mm}$. Of the 14 patients, three and two patients exhibited N1a (21.4\%) and T3 $(14.3 \%)$ stages of cancer, respectively.

The presumed costs of screening thyroid US and US-guided FNA (including the cost of cytopathological interpretation) in South Korea are $\$ 100$ and $\$ 250$, respectively. The total costs for US and FNA in this study were $\$ 184,500(\$ 100 \times 1,845)$ and $\$ 27,750(\$ 250 \times 111)$, respectively. The estimated average cost per patient for the diagnosis of cancer in this study was $\$ 7,319[(\$ 184,500+\$ 27,750) / 29]$.

\section{Discussion}

In the present study, screening thyroid US revealed no significant differences in the rate of malignancy of thyroid nodules among patients according to age or sex, which is similar to the results of previous screening studies $[15,16]$. The incidence of thyroid cancer is known to be relatively high among women, and age is an important prognostic factor of mortality in patients with thyroid cancer $[16,20]$. The discrepancy between these facts and our findings may be explained by the fact that the population screened in the present study, which mainly comprised self-referred subjects of higher economic status than the general population, might not be representative of the general population. In addition, screening US exhibited good diagnostic performance, and the US features of screening-detected thyroid cancer were found to be significantly different from those of benign nodules and concordant with those reported in previous studies [15]. The significant US findings of screening-detected cancer observed in the present study were similar to those specified by the Korean and American guidelines on malignant US features for thyroid nodules [18,21].

The prevalence of thyroid incidentalomas in the present study $(64.2 \%)$ is similar to that reported by Russ et al. [22], who found that $70 \%-83 \%$ of the general population exhibited small thyroid nodules detectable with US. The rate of malignancy observed in our study (2.4\%) was slightly lower than those reported in previous studies $[16,20,23]$. This discrepancy might be attributable to differences in the inclusion criteria of thyroid incidentaloma and the indications for US-guided FNA among studies. In the present study, we included all thyroid nodules identified by US, regardless of size, and US-guided FNA was performed when essential according to the KSThR guidelines [18]. In addition, the incidence of diffuse thyroiditis on thyroid US in this study was 7\%. It is occasionally difficult to differentiate diffuse thyroiditis and nodules on thyroid US. In a previous reference, Kim et al. [24] reported that real-time US performed by an experienced radiologist was useful to differentiate patients with asymptomatic diffuse thyroid disease from those with normal thyroid. As is the case of the normal parenchyma, differentiation of thyroid nodules from thyroiditis would not be difficult for an experienced radiologist. In the present study, thyroid US examination and image analysis for all subjects was achieved by experienced radiologists; therefore, the results of this study are reliable.

The rate of cancer detection in this study, which was defined as the total number of screening-detected cancers per total number of screening US examinations, was 1.6\% (15.7 thyroid cancers detected per 1,000 examinations). Previous studies involving medical screening of populations have 
reported $0.95-2.9 \%$ rates of detection of thyroid cancer $[15,16]$. We predicted the approximate cost of thyroid US and US-guided FNA in South Korea to be $\$ 184,500(\$ 100 \times 1,845)$ and $\$ 27,750(\$ 250 \times 111)$, respectively, which implies that an average sum of $\$ 7,319[(\$ 184,500+\$ 27,750) / 29]$ was paid for each case of cancer diagnosis. To the best of our knowledge, there is no previous reference of social cost and cost-analysis of screening-detected thyroid cancer in the literature. The Government of South Korea provides National Health Insurance Service, which includes screening for several types of cancers, including stomach, large intestine, liver, and breast cancers. Although thyroid cancer is not included in this system, the number of thyroid US examinations has increased because of the demand for US screening by self-referred patients. With regard to breast cancer screening, the rate of detection of cancer during routine mammography was reported to range from 3.0 to 7.9 cases of cancer per 1,000 examinations $[25,26]$. Upon comparison with the results of our study, it is evident that the rate of detection of cancer by thyroid screening US is 2-5 times higher than that obtained by screening mammography. The biological behavior and disease progression of these two types of cancers differ, with breast cancer having a worse prognosis than thyroid cancer, and the latter showing a relatively benign or indolent course of disease progression, especially in occult cases $[27,28]$. In addition, there are a limited number of references regarding the cost-analysis of screening US relative to the decrease in mortality and morbidity rates. Thus, considering the indolent nature of thyroid cancer, we believe that long-term randomized controlled trials must be performed in the future to investigate the efficacy of screening thyroid US.

In the present study, 18 patients finally underwent thyroid surgery at our institution, 14 (77.8\%) of whom had thyroid microcarcinoma, which was defined by nodules with longitudinal diameter $<10 \mathrm{~mm}$. About $17 \%$ of operative patients and $14 \%$ of patients with microcarcinoma exhibited T3 stage cancer, with extrathyroidal extension and capsular invasion. Patients included in our study exhibited lower tumor and nodal pathological stages of cancer than those reported in a previous study $[16,29]$. However, management of thyroid microcarcinoma is a controversial subject $[16,29,30]$, and the sample size of operative patients in the present study might be too small to arrive at a firm conclusion regarding the best management practice.

This study has several limitations, the first being the small sample size and retrospective nature of the study. In particular, the total number of cases of screening-detected cancer was smaller than the number of benign cases. However, according to the rate of malignancy reported in a previous study, our result is within the acceptable range $[16,20,23]$. Second, since US-guided FNA was selectively performed for indeterminate and probably benign nodules according to the KSThR guidelines, the diagnoses of the rest of the cases were not pathologically confirmed. The possibility of false-negative results in these cases cannot be excluded. However, patients were followed-up for an adequate period of at least 1 year to minimize the possibility of false-negative results. Third, our estimated social cost of screening thyroid US was evaluated based on the clinical setting in South Korea, and therefore might vary from that in other countries. As a result, the cost-analysis of screening thyroid US projected in our study cannot be generalized to the rest of the world. In addition, our purpose was to calculate the social cost of diagnosis of thyroid cancer in an asymptomatic population; therefore, we only analyzed the cost of diagnosis, while other parameters such as costs associated with surgeries or potential complications were not considered. Finally, the diagnostic performance of screening thyroid US for malignancies was calculated based on US findings. Because we evaluated thyroid cancers visualized on US images by radiologists, the diagnostic performance might be underestimated owing to undetected small cancers or cancers that were overlooked.

In conclusion, screening thyroid US exhibited a good diagnostic performance and demonstrated significant differences in US features between screening-detected cancer and benign nodules. Further prospective studies, including randomized controlled trials, must be conducted to evaluate the exact influence of screening thyroid US considering the indolent progression of the disease.

\section{Conflicts of Interest}

Conflict of interest relevant to this article was not reported.

\section{References}

1. Vander JB, Gaston EA, Dawber TR. The significance of nontoxic thyroid nodules. Final report of a 15-year study of the incidence of thyroid malignancy. Ann Intern Med. 1968;69: 537-40.
2. Ezzat S, Sarti DA, Cain DR, Braunstein GD. Thyroid incidentalomas: prevalencee by palpation and ultrasonography. Arch Intern Med. 1994;154:1838-40.

3. Mortensen JD, Woolner LB, Bennett WA. Gross and micro- 
scopic findings in clinically normal thyroid glands. J Clin Endocrinol Metab. 1955;15:1270-80.

4. Watters DA, Ahuja AT, Evans RM, Chick W, King WW, Metreweli C, et al. Role of ultrasound in the management of thyroid nodules. Am J Surg. 1992;164:654-7.

5. Steele SR, Martin MJ, Mullenix PS, Azarow KS, Andersen CA. The significance of incidental thyroid abnormalities identified during carotid duplex ultrasonography. Arch Surg. 2005;140: 981-5.

6. Frates MC, Benson CB, Charboneau JW, Cibas ES, Clark OH, Coleman BG, et al. Management of thyroid nodules detected at US: Society of Radiologists in Ultrasound consensus conference statement. Radiology. 2005;237:794-800.

7. Kilfoy BA, Zheng T, Holford TR, Han X, Ward MH, Sjodin A, et al. International patterns and trends in thyroid cancer incidence, 1973-2002. Cancer Causes Control. 2009;20:525-31.

8. Davies L, Welch HG. Increasing incidence of thyroid cancer in the United States, 1973-2002. JAMA. 2006;295:2164-7.

9. Ahn HS, Kim HJ, Welch HG. Korea's thyroid-cancer "epidemic": screening and overdiagnosis. N Engl J Med. 2014; 371:1765-7.

10. Chen AY, Jemal A, Ward EM. Increasing incidence of differentiated thyroid cancer in the United States, 1988-2005. Cancer. 2009;115:3801-7.

11. Morris LG, Myssiorek D. Improved detection does not fully explain the rising incidence of well-differentiated thyroid cancer: a population-based analysis. Am J Surg. 2010;200:454-61.

12. Wartofsky L. Increasing world incidence of thyroid cancer: increased detection or higher radiation exposure? Hormones (Athens). 2010;9:103-8.

13. Jung KW, Won YJ, Kong HJ, Oh CM, Cho H, Lee DH, et al. Cancer statistics in Korea: incidence, mortality, survival, and prevalence in 2012. Cancer Res Treat. 2015;47:127-41.

14. Yi KH, Kim SY, Kim DH, Kim SW, Na DG, Lee YJ, et al. The Korean guideline for thyroid cancer screening. J Korean Med Assoc. 2015;58:302-12.

15. Kim JY, Lee CH, Kim SY, Jeon WK, Kang JH, An SK, et al. Radiologic and pathologic findings of nonpalpable thyroid carcinomas detected by ultrasonography in a medical screening center. J Ultrasound Med. 2008;27:215-23.

16. Choi YJ, Park YL, Koh JH. Prevalence of thyroid cancer at a medical screening center: pathological features of screendetected thyroid carcinomas. Yonsei Med J. 2008;49:748-56.

17. Liel Y. Screening without evidence of efficacy: thyroid ultrasonography is another example. BMJ. 2004;328:521.

18. Moon WJ, Baek JH, Jung SL, Kim DW, Kim EK, Kim JY, et al. Ultrasonography and the ultrasound-based management of thyroid nodules: consensus statement and recommendations. Korean J Radiol. 2011;12:1-14.

19. Cibas ES, Ali SZ; NCI Thyroid FNA State of the Science Conference. The Bethesda System For Reporting Thyroid Cytopathology. Am J Clin Pathol. 2009;132:658-65.

20. Kang HW, No JH, Chung JH, Min YK, Lee MS, Lee MK, et al. Prevalence, clinical and ultrasonographic characteristics of thyroid incidentalomas. Thyroid. 2004;14:29-33.

21. Haugen BR, Alexander EK, Bible KC, Doherty GM, Mandel SJ, Nikiforov YE, et al. 2015 American Thyroid Association management guidelines for adult patients with thyroid nodules and differentiated thyroid cancer: The American Thyroid Association Guidelines Task Force on Thyroid Nodules and Differentiated Thyroid Cancer. Thyroid. 2016;26:1-133.

22. Russ G, Leboulleux S, Leenhardt L, Hegedus L. Thyroid incidentalomas: epidemiology, risk stratification with ultrasound and workup. Eur Thyroid J. 2014;3:154-63.

23. Nam-Goong IS, Kim HY, Gong G, Lee HK, Hong SJ, Kim WB, et al. Ultrasonography-guided fine-needle aspiration of thyroid incidentaloma: correlation with pathological findings. Clin Endocrinol (Oxf). 2004;60:21-8.

24. Kim DW, Eun CK, In HS, Kim MH, Jung SJ, Bae SK. Sonographic differentiation of asymptomatic diffuse thyroid disease from normal thyroid: a prospective study. AJNR Am J Neuroradiol. 2010;31:1956-60.

25. Berg WA, Blume JD, Cormack JB, Mendelson EB, Lehrer D, Bohm-Velez M, et al. Combined screening with ultrasound and mammography vs mammography alone in women at elevated risk of breast cancer. JAMA. 2008;299:2151-63.

26. Moon HJ, Jung I, Park SJ, Kim MJ, Youk JH, Kim EK. Comparison of cancer yields and diagnostic performance of screening mammography vs. supplemental screening ultrasound in 4394 women with average risk for breast cancer. Ultraschall Med. 2015;36:255-63.

27. Solares CA, Penalonzo MA, Xu M, Orellana E. Occult papillary thyroid carcinoma in postmortem species: prevalence at autopsy. Am J Otolaryngol. 2005;26:87-90.

28. Neuhold N, Kaiser H, Kaserer K. Latent carcinoma of the thyroid in Austria: a systematic autopsy study. Endocr Pathol. 2001;12:23-31.

29. Lee J, Rhee Y, Lee S, Ahn CW, Cha BS, Kim KR, et al. Frequent, aggressive behaviors of thyroid microcarcinomas in korean patients. Endocr J. 2006;53:627-32.

30. Ito Y, Uruno T, Nakano K, Takamura Y, Miya A, Kobayashi $\mathrm{K}$, et al. An observation trial without surgical treatment in patients with papillary microcarcinoma of the thyroid. Thyroid. 2003;13:381-7. 